\title{
Effect of an Anterior Cruciate Ligament Rupture on Knee Proprioception Within 2 Years After Conservative and Operative Treatment: A Systematic Review with Meta-Analysis
}

\author{
John Dick Fleming ${ }^{1} \cdot$ Ramona Ritzmann ${ }^{2} \cdot$ Christoph Centner $^{1,2}$ (D)
}

Accepted: 5 November 2021 / Published online: 2 December 2021

(c) The Author(s) 2021

\begin{abstract}
Background The anterior cruciate ligament (ACL) plays a major role in knee proprioception and is thus responsible for maintaining knee joint stability and functionality. The available evidence suggests that ACL reconstruction diminishes somatosensory feedback and proprioceptive functioning, which are vital for adequate joint positioning and movement control. Objective The aim of this systematic review and meta-analysis was to investigate the effect of an ACL rupture on knee proprioception after arthroscopic ACL repair surgery or conservative treatment.

Methods A systematic review with meta-analysis was conducted according to the Preferred Reporting Guidelines for Systematic Reviews and Meta-Analyses (PRISMA) guidelines. The literature search was performed in the following databases from inception to 10th October 2020: PubMed, Web of Science, SPORTDiscus, Cochrane Library and Scopus. Randomized and non-randomized studies that evaluated proprioception using the joint position sense (JPS) and threshold to detection of passive motion (TTDPM) techniques at $15^{\circ}-30^{\circ}$ knee flexion with an external healthy control group in a time period between 6 and 24 months post injury or operation were included in the analysis.

Results In total, 4857 studies were identified, from which 11 were included in the final quantitative analysis. The results demonstrated that proprioception after arthroscopic ACL repair surgery was significantly lower than in the healthy control group (JPS: standardized mean difference [SMD] 0.57, 95\% confidence interval [CI] $0.27-0.87, p<0.01, n=6$ studies; TTDPM: SMD $0.77,95 \%$ CI $0.20-1.34, p<0.01, n=4$ studies). There were no significant differences in proprioception between the conservative treatment group and the healthy control group (JPS: SMD 0.57, 95\% CI -0.69 to $1.84, p=0.37$, $n=4$ studies; TTDPM: SMD $0.82,95 \%$ CI -0.02 to $1.65, p=0.05, n=2$ studies), although measures for TTDPM were close to statistical significance.

Conclusion The findings of the present systematic review and meta-analysis revealed that knee proprioception is persistently compromised 6-24 months following surgical treatment of ACL tears compared with healthy controls. The reduced kinesthetic awareness after ACL surgery is of high relevance for optimizing individual treatment plans in these patients. As the current literature is still scarce about the exact underlying mechanisms, further research is needed.
\end{abstract}

Trial Registration The present systematic review was registered in PROSPERO (CRD42021198617).

Christoph Centner

Christoph.centner@sport.uni-freiburg.de

1 Department of Sport and Sport Science, University of Freiburg, Schwarzwaldstraße 175, 79117 Freiburg,

Germany

2 Praxisklinik Rennbahn, Muttenz, Switzerland 


\section{Key Points}

We found high-level evidence suggesting that proprioceptive deficits are present following surgical anterior cruciate ligament reconstruction.

Although no significant deficits were detected following conservative approaches, we observed considerable heterogeneity between these studies that needs to be taken into account when interpreting the findings for conservative treatments.

\section{Introduction}

The anterior cruciate ligament (ACL) plays a major role in maintaining knee joint stability because it contributes to both functionality and the mechanical congruence of the lateral and medial tibiofemoral joints. Based on its anatomical position, it resists the anterior tibial translation and rotational load [1]. Furthermore, knee ligaments are rich in sensory innervation, which allows them to be closely integrated in neural reflex pathways [2,3]. Throughout abnormal strain, stimulation of mechanoreceptors in the ACL initiates different types of reflex responses through the neural arc to secure the arthrokinematics of the joint with an adequate muscle contraction $[4,5]$.

In sports, an ACL tear is one of the most common injuries, occurring mainly in pivoting high-load sports such as soccer, basketball and alpine ski [1]. The main focus of either an operative or conservative treatment and the following rehabilitation program is to therefore restore the stability and kinematics of the joint to ensure a safe return to sport. Nevertheless, studies indicate that of $82 \%$ of the patients who return to sport, only $63 \%$ compete at their original level of competition [6]. This is accompanied by an increased risk of recurrences in the first 2 years post injury [7] and the long-term consequences of developing knee osteoarthritis [8].

One cause of the increased risk of re-injury and performance declines is attributed to the diminished proprioception after an ACL rupture [9]. The native intact ACL contains mechanoreceptors that detect changes in direction of movement, changes in acceleration, speed, tension and an estimate of the joint position $[10,11]$. The ACL does not heal when torn, and surgical reconstruction after a complete ACL tear using tendinous allografts or autografts does not allow for the re-innervation of mechanoreceptors [12]. A key factor in persistent functional instability after ACL tears is therefore experiencing kinesthetic deficiency and altered neuromuscular function secondary to a diminished somatosensory feedback $[10,11]$. Proprioceptive information to accurately regulate the neuromuscular control is missing and may account for an increased risk of re-injury and coordination deficits when high performance is required. Methodological approaches to assess proprioception include the detection and reproduction of angular position, sense of tension, or effort [13]. Generally, the two most common protocols to reliably assess knee proprioception are the joint position sense (JPS) and threshold to detect passive motion (TTDPM) measures [14, 15]. JPS is assessed by measuring the reproduction of passive angular positioning [15]. TTDPM evaluates the ability of individuals to detect the onset of passive movement [16].

Although older previous systematic reviews exist [17, 18], which aimed to elucidate the effects of ACL injury on knee proprioception, both reviews included heterogeneous studies that limit the validity and significance of their conclusions. Heterogeneity arose from comparing studies that assessed broad time points extending from 8 to 60 months following ACL injury [18] or that focused on paradigms with different joint angles (e.g., $15^{\circ}$ vs. $75^{\circ}$ knee flexion [17]) in which ligamentous tension is not necessarily experienced and kinaesthesia is of negligible importance [19, 20]. Lastly, Relph and colleagues [17] included single study populations multiple times in their quantitative analyses, which inherently increases the statistical weighting of that specific population and thus potentially biases the results.

Against this background and the fact that numerous studies have recently been published on this topic, it is highly relevant to systematically re-investigate the effects of ACL injury on proprioception with strict inclusion criteria in order to ensure homogeneity between studies and thus allow a conclusive statement of clinical relevance. For that purpose, the present systematic review and meta-analysis aims to systematically consider several biasing factors, such as different timepoint assessments, various knee angle measurements, and the use of different autografts and allografts within the methodological approach. Subsequently, the clinical relevance will be determined by interpreting the calculated effect sizes.

\section{Methods}

\subsection{Protocol and Registration}

The present systematic review includes a meta-analytic approach and was completed in accordance with the Preferred Reporting Items for Systematic Reviews and MetaAnalyses (PRISMA) guidelines [21]. Therefore, a review 
protocol was prospectively elaborated and registered on PROSPERO (CRD42021198617).

\subsection{Search Strategy}

To identify relevant studies, a literature search was performed in the following electronic databases from inception to 10 October 2020: PubMed, Web of Science, Scopus, SPORTDiscus and Cochrane Library. The search string consisted of synonyms from the field of ACL injury connected with synonyms for the topic of proprioception. Databases were searched without restrictions ("All field" search) and the final search string was:

("Joint Position Sense" OR "TTDPM" OR Proprioception OR active angle reproduction OR Threshold to detect passive motion $O R$ passive angle reproduction $O R$ kinesthes* OR somatosensory* OR mechanorecept* ${ }^{*}$ OR "balance") AND ("Anterior cruciate ligament" $O R$ "ACL rupture" OR "ACL reconstruction" OR "ACL injury" $O R$ "ACL deficient" OR "knee injury" OR "knee joint" OR "ACL replacement").

Additionally, the reference lists of eligible articles were systematically screened for further eligible papers [22]. All studies including title and abstract were exported to a citation manager and duplicates were removed before further processing (for the search process, see Fig. 1).

\subsection{Study Selection and Eligibility Criteria}

To assess eligibility, two reviewers independently evaluated the results by screening the title and abstracts. In cases of relevant titles, the full text was assessed. The inclusion criteria included (1) complete primary ACL rupture; (2) no additional knee pathology; (3) the therapeutic treatment included either surgery or a conservative approach; and (4) comparison with a healthy control (HC) group. Comparing against HCs is crucial given that evidence suggests that the proprioception of the contralateral intact knee can also be compromised following an ACL tear [18, 23]. Re-ruptures and incomplete ruptures were excluded to ensure an homogenous population with comparable sensorimotor prerequisites. Lastly, only studies with proprioception assessments between $0^{\circ}$ and $30^{\circ}$ knee angle were included in order to avoid inhomogeneity of studies. The reason for focusing on this angle range was that the ACL is subjected to the highest stress in terminal knee positions [24, 25].

Studies were excluded if their study design incorporated a specific treatment (e.g., knee orthosis, proprioceptive training, etc.). Furthermore, studies were not included if proprioception was measured later than 24 months post trauma, since this has been postulated to be an important time span for return to sport and increased re-injury rates [7, 26, 27]. Finally, studies with weak quality (score $\leq 14$ on the Downs and Black Checklist [28]) were excluded from the qualitative and quantitative analyses.

The identified studies were subsequently grouped regarding treatment (operative vs. conservative) and quantitative analyses were conducted considering the reliable and accurate measurement techniques of proprioception: JPS and TTDPM.

\subsection{Data Extraction and Collection}

After an initial screening, the following information was extracted from relevant articles: (1) population characteristics; (2) measurement timepoints; (3) methodological approach; and (4) main findings.

Fig. 1 Study selection process

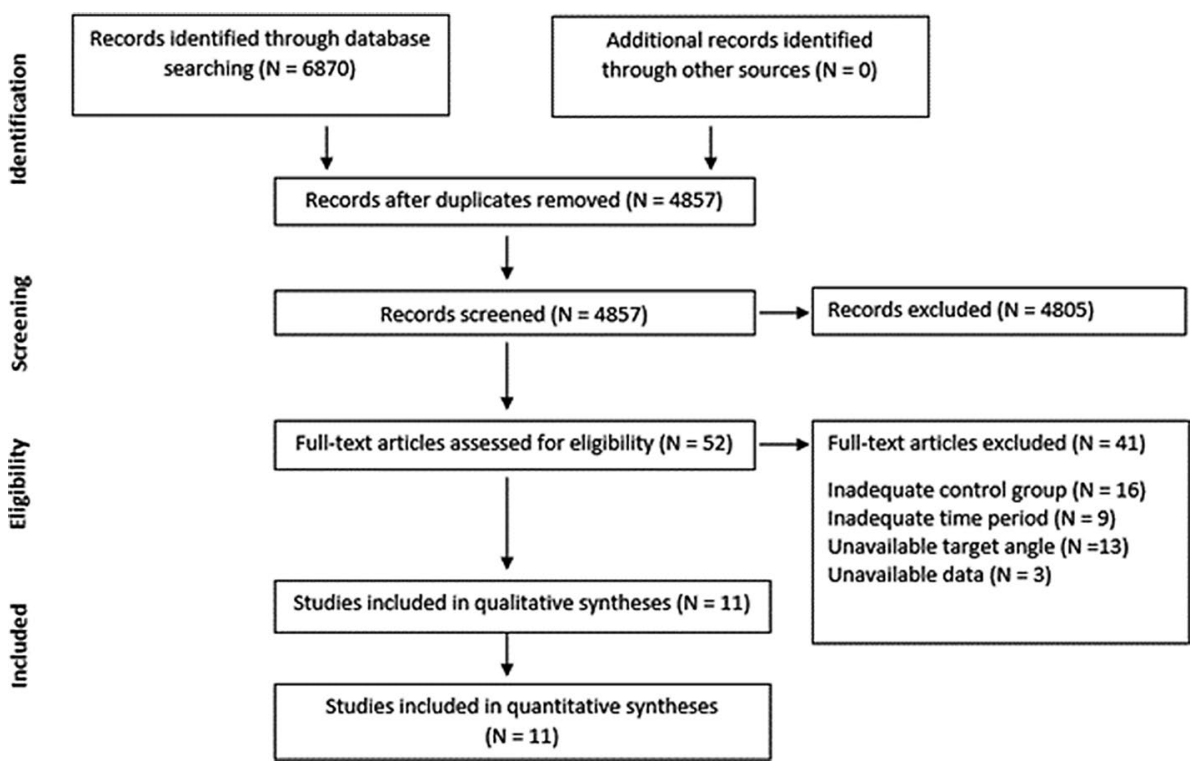


During data extraction, only timepoints within the first 24 months following the ACL injury were considered. Since some studies reported several timepoints within this period, an a priori prioritization list was developed $[29,30]$ and the timepoints were considered in the following order: $24 \mathrm{mont}$ hs $>12$ months $>6$ months $>3$ months. Additionally, target angles during proprioception measurements were prioritized from $15^{\circ}$ to $30^{\circ}$. The reason for this prioritization was that the injury mechanism has previously been associated with a knee angle around $15^{\circ}$ [31-33]. Furthermore, measurements in the direction of knee extension were considered first compared with flexion, when measurements in both directions were available. If only knee flexion values were available, these were also included in the analysis. Lastly, if both allograft and autografts were included, autografts were prioritized since these are more frequently used in ACL surgery [1]. Within autografts, the following order was chosen: (1) bone-to-bone patellar tendon autograft; (2) semitendinosus tendon autograft; (3) quadriceps tendons autograft.

In cases of unavailable raw data, the corresponding author of the manuscript was contacted. If the respective authors were non-responsive, data were extrapolated from figures using ImageJ software (National Institutes of Health, Bethesda, MD, USA). If extrapolation from figures was not possible, data were discussed within the qualitative analyses but not within the quantitative evaluation.

All data were independently extracted and screened by two researchers (JF and CC). In the case of disagreement, consensus was found in one of the regular discussion meetings.

\subsection{Study Quality}

Study quality was assessed using the Downs and Black Checklist $[28,34]$. The checklist is used to evaluate randomized and non-randomized studies and consists of 27 items covering the following quality characteristics: reporting, external validity, internal validity (bias and confounding variables) and power. The total score is categorized as follows: very good (26-28 points), good (20-25 points), moderate (15-19 points), or weak ( $0-14$ points) [34].

\subsection{Risk-of-Bias Assessment}

Risk of bias within each study was determined using the JBI Critical Appraisal Checklist for analytical cross-sectional trials [35-37]. This tool comprises a total of eight items and two researchers (JF and CC) independently rated each item as 'yes', 'no', 'unclear' or 'not applicable' as per protocol [35]. The studies were rated as low risk of bias if more than $70 \%$ of the items were applicable ('yes' answer). In cases of 50-69\% applicable items, a moderate risk of bias was assumed, and with $<49 \%$ applicable items, a high risk of bias was assumed [38].

\subsection{Synthesis of Results and Statistical Approach}

For the quantitative meta-analytical combination, the standardized mean difference (SMD) was calculated from the primary studies. The SMD was computed by dividing the mean difference by the pooled standard deviation [39]. Therefore, mean, sample size, and standard deviation were extracted. If mean or standard deviation from a study were not reported, these were respectively estimated from the median or standard error and confidence intervals (CIs) according to the Cochrane Handbook of Systematic Reviews [39]. All metaanalyses were performed using a random-effects model with inverse variance weighting, and forest plots were subsequently created. To examine inconsistency and heterogeneity across studies, the $I$-square method was used. $I$-square was calculated as [(Chi-square statistic - degrees of freedom)/ Chi-square statistic $\times 100 \%$ ] [39]. With respect to previous interpretation guidelines [39], $I$-square was interpreted as follows: 0-40\% representing low heterogeneity, 30-60\% representing moderate heterogeneity, $50-90 \%$ representing substantial heterogeneity, and $75-100 \%$ representing considerable heterogeneity. Lastly, a qualitative analysis of proprioception following ACL reconstructed (ACLR) and ACL deficient (ACLD) was conducted taking the different methodologies of proprioception assessment into account.

\section{Results}

\subsection{Study Selection}

In total, 4857 studies were identified throughout the literature search, with 11 finally included in this systematic review with meta-analysis. We assessed the full-texts from 52 studies, from which 41 were excluded due to an inadequate control group $(n=16)$, inadequate time period $(n=9)$, unavailable target angle $(n=13)$, or unavailable data $(n=3)$. The remaining 11 studies were then assigned to their treatment (operative or conservative) and, for quantitative analyses, again divided (and independently analyzed) into studies using JPS and TTDPM as measures of proprioception.

\subsection{Study Characteristics}

Within the included studies comparing patients with a medical history of surgical ACL reconstruction with HCs, six studies quantified knee proprioception using JPS [40-45] and four studies by means of TTDPM [41, 42, 46, 47]. In three studies, JPS was estimated in a seating position [40, 44, 45]; three studies evaluated JPS in a standing [43], supine 
[42], or lateral recumbent position [41], respectively. Similarly, TTDPM was measured in the seated [46, 47], supine [42], and lateral recumbent positions [41]. The mean time between injury and assessment (which was included in the present quantitative analysis) in the comparison between ACLR and HCs was 14 months (see Tables 1 and 2).

Within studies comparing ACL-deficient patients and HCs, four studies assessed JPS [40, 48-50] and two studies assessed TTDPM [46, 48]. Positions for JPS included the seated [40, 49, 50] and lateral recumbent positions [48]. Studies using TTDPM investigated knee proprioception, also in the seated [46] and lateral recumbent positions [48] (Tables 3 and 4).

\subsection{Anterior Cruciate Ligament Reconstructed (ACLR) Versus Healthy Controls (HCs)}

The results of the ACLR group compared with the HC group revealed significant differences in both measures for proprioception (JPS and TTDPM).
In total, six studies evaluated JPS and four studies analyzed TTDPM. The analysis for the JPS demonstrated an SMD of 0.57 (95\% CI 0.27-0.87), favoring the HC group (Fig. 2). On average, the mean angle of error was therefore significantly higher for the ACLR group compared with the $\operatorname{HC}$ group $(Z=3.75, p<0.01)$. Study heterogeneity was low $\left(I^{2}=14 \%\right)$ and not statistically significant $(p=0.33)$.

For the four studies analyzing the TTDPM, an SMD of 0.77 (95\% CI $0.20-1.34$ ), also favoring the HC group, was identified (Fig. 3). The TTDPM was also significantly higher in the ACLR group compared with the control group $(Z=2.64, p<0.01) . I$-squared demonstrated a substantial study heterogeneity with a value of $65 \%(p<0.05)$.

\subsection{Anterior Cruciate Ligament Deficient (ACLD) versus HCs}

Across four studies that assessed differences in JPS between ACLD and HC, an SMD of 0.57 (95\% CI - 0.69 to 1.84) was identified, which was not statistically significant $(Z=0.89$,

Table 1 Study characteristics of the ACLR group-JPS $(n=6)$

\begin{tabular}{|c|c|c|c|c|}
\hline Study & Groups & Months after injury & JPS set-up & Starting and target angle \\
\hline Fremerey et al. [40] & $\begin{array}{l}\mathrm{ACLR}=20 \\
\mathrm{HC}=20\end{array}$ & 3,6 & $\begin{array}{l}\text { PAR } \\
\text { Seated } \\
0.5^{\circ} / \mathrm{s}\end{array}$ & $\begin{array}{l}\text { Start at } 0^{\circ} \mathrm{TF} \\
\text { Target angle: } 0-20^{\circ}, 40-60^{\circ}, 80-100^{\circ}\end{array}$ \\
\hline Roberts et al. [41] & $\begin{array}{l}\mathrm{ACLR}=20 \\
\mathrm{HC}=19\end{array}$ & 24 & $\begin{array}{l}\text { AAR } \\
\text { Lateral recumbent position }\end{array}$ & $\begin{array}{l}\text { Start at } 30^{\circ} \mathrm{TF} \text { and } 60^{\circ} \mathrm{TE} \\
\text { Target angle: } 60^{\circ}(\mathrm{TF}) \text { and } 30^{\circ}(\mathrm{TE})\end{array}$ \\
\hline Bonfim et al. [42] & $\begin{array}{l}\mathrm{ACLR}=10 \\
\mathrm{HC}=10\end{array}$ & 18 & $\begin{array}{l}\text { Verbal, external goniometer } \\
\text { Supine position }\end{array}$ & $\begin{array}{l}\text { Start at } 0^{\circ} \mathrm{TF} \\
\text { Target angle: } 0^{\circ}, 15^{\circ}, 30^{\circ}, 45^{\circ}, 60^{\circ}\end{array}$ \\
\hline Mir et al. [43] & $\begin{array}{l}\mathrm{ACLR}=12 \\
\mathrm{HC}=12\end{array}$ & 11 & $\begin{array}{l}\text { AAR } \\
\text { Standing }\end{array}$ & $\begin{array}{l}\text { Start at } 0^{\circ} \mathrm{TF} \text { and } 60^{\circ} \mathrm{TE} \\
\text { Target angle: } 30^{\circ}\end{array}$ \\
\hline Zhou et al. [44] & $\begin{array}{l}\mathrm{ACLR}=36 \\
\mathrm{HC}=13\end{array}$ & 6 & $\begin{array}{l}\text { PAR } \\
\text { Seated } \\
2 \% / s\end{array}$ & $\begin{array}{l}\text { Start at } 0^{\circ} \mathrm{TF} \\
\text { Target angle: } 0-20^{\circ}, 40-60^{\circ}, 80-100^{\circ}\end{array}$ \\
\hline San Martín-Mohr et al. [45] & $\begin{array}{l}\mathrm{ACLR}=30 \\
\mathrm{HC}=27\end{array}$ & $7.77 \pm 2.28$ & $\begin{array}{l}\text { AAR } \\
\text { Seated }\end{array}$ & $\begin{array}{l}\text { Start at } 90^{\circ} \mathrm{TE} \\
\text { Target angle: } 0-30^{\circ}, 30-60^{\circ}, 60-90^{\circ}\end{array}$ \\
\hline
\end{tabular}

$A C L R$ anterior cruciate ligament reconstructed, $H C$ healthy control, JPS joint position sense, $P A R$ passive angle reproduction, $A A R$ active angle reproduction, $T F$ towards flexion, $T E$ towards extension

Table 2 Study characteristics of the ACLR group-TTDPM $(n=4)$

\begin{tabular}{|c|c|c|c|c|}
\hline Study & Groups & Months after injury & TTDPM set-up & Starting angle and direction of movement \\
\hline Roberts et al. [41] & $\begin{array}{l}\mathrm{ACLR}=20 \\
\mathrm{HC}=19\end{array}$ & 24 & $\begin{array}{l}\text { Lateral recumbent position } \\
0.5 \% \mathrm{~s}\end{array}$ & Start at $20^{\circ}$ and $40^{\circ} \mathrm{TF}$ and $\mathrm{TE}$ \\
\hline Bonfim et al. [42] & $\begin{array}{l}\mathrm{ACLR}=10 \\
\mathrm{HC}=10\end{array}$ & 18 & $\begin{array}{l}\text { Supine position } \\
0.5 \% \mathrm{~s}\end{array}$ & $\begin{array}{l}\text { Start TF at } 0^{\circ}, 15^{\circ}, 30^{\circ}, 45^{\circ}, 60^{\circ} \\
\text { Start TE at } 15^{\circ}, 30^{\circ}, 45^{\circ}, 60^{\circ}\end{array}$ \\
\hline Ozenci et al. [46] & $\begin{array}{l}\mathrm{ACLR}=20 \\
\mathrm{HC}=20\end{array}$ & $16.5 \pm 5.5$ & $\begin{array}{l}\text { Seated } \\
1 \% \mathrm{~s}\end{array}$ & Start at $15^{\circ} \mathrm{TE}$ and TF \\
\hline Laboute et al. [47] & $\begin{array}{l}\mathrm{ACLR}=32 \\
\mathrm{HC}=32\end{array}$ & 6 & $\begin{array}{l}\text { Seated } \\
4 \%\end{array}$ & Start at $15^{\circ} \mathrm{TF}$ \\
\hline
\end{tabular}

$A C L R$ anterior cruciate ligament reconstructed, $H C$ healthy control, TTDPM threshold to determine passive motion, $T F$ towards flexion, $T E$ towards extension 
Table 3 Study characteristics of the ACLD group-JPS $(n=4)$

\begin{tabular}{lllll}
\hline Study & Groups & Months after injury & JPS set-up & Starting and target angle \\
\hline Fridén et al. [48] & ACLD $=16$ & $1,2,4,8$ & $\begin{array}{l}\text { AAR } \\
\text { Lateral recumbent } \\
\text { position } \\
\text { PC }=19\end{array}$ & $\begin{array}{l}\text { Start at } 30^{\circ} \mathrm{TF} \text { and } 60^{\circ} \mathrm{TE} \\
\text { Target angle: } 30^{\circ}\end{array}$ \\
Fremerey et al. [40] & $\mathrm{ACLD}=20$ & $12.4 \pm 3.7$ & $\begin{array}{l}\text { PAR } \\
\text { Seated }\end{array}$ & $\begin{array}{l}\text { Start at } 0^{\circ} \mathrm{TF} \\
\text { Target angle: } 0-20^{\circ}, 40-60^{\circ}, 80-100^{\circ}\end{array}$ \\
& $\mathrm{HC}=20$ & & AAR & Start at $0^{\circ} \mathrm{TF}$ \\
Relph and Herrington [49] & $\mathrm{ACLD}=20$ & $11 \pm 2$ & Seated & Target angle: $10-30^{\circ}$ \\
Zult et al. [50] & $\mathrm{HC}=20$ & & AAR & Start at $90^{\circ} \mathrm{TE}$ \\
& $\mathrm{ACLD}=32$ & 7 & Seated & Target angle: $15^{\circ}, 30^{\circ}, 45^{\circ}, 60^{\circ}$ \\
\hline
\end{tabular}

$A C L D$ anterior cruciate ligament deficient, $H C$ healthy control, JPS joint position sense, PAR passive angle reproduction, $A A R$ active angle reproduction, $T F$ towards flexion, $T E$ towards extension

Table 4 Study characteristics of the ACLD group-TTDPM $(n=2)$

\begin{tabular}{|c|c|c|c|c|}
\hline Study & Groups & Months after injury & TTDPM set-up & $\begin{array}{l}\text { Starting angle and } \\
\text { direction of movement }\end{array}$ \\
\hline Fridén et al. [48] & $\begin{array}{l}\mathrm{ACLD}=16 \\
\mathrm{HC}=19\end{array}$ & $1,2,4,8$ & $\begin{array}{l}\text { Lateral recumbent } \\
\text { position } \\
0.5 \% \mathrm{~s}\end{array}$ & $\begin{array}{l}\text { Start at } 20^{\circ} \mathrm{TE} \text { and TF } \\
\text { Start at } 40^{\circ} \mathrm{TE} \text { and TF }\end{array}$ \\
\hline Ozenci et al. [46] & $\begin{array}{l}\mathrm{ACLD}=20 \\
\mathrm{HC}=20\end{array}$ & $12.5 \pm 3.6$ & $\begin{array}{l}\text { Seated } \\
1 \% \mathrm{~s}\end{array}$ & Start at $15^{\circ} \mathrm{TE}$ and $\mathrm{TF}$ \\
\hline
\end{tabular}

$A C L D$ anterior cruciate ligament deficient, $H C$ healthy control, TTDPM threshold to determine passive motion, $T F$ towards flexion, $T E$ towards extension $p=0.37)$. In this comparison, considerable study heterogeneity was demonstrated $\left(I^{2}=93 \%, p<0.01\right)$ (Fig. 4$)$.

An effect size of 0.82 (95\% CI -0.02 to 1.65 ) was calculated for studies investigating TTDPM. Similarly, no significant difference between the two groups (ACLD vs. HCs) could be found $(Z=1.92 ; p=0.05)$. The study heterogeneity with an $I$-square of $67 \%$ was substantial $(p=0.08)$ (Fig. 5).

\subsection{Quality Assessment}

Table 5 shows the final quality scores of all included studies, and the mean score. The included studies had a mean quality score of 19 on the Downs and Black Checklist. According to Silverman et al. [34], a score of 19 indicates a sufficient and fair study quality [34].

\subsection{Risk of Bias}

The assessment of the risk of bias revealed an overall low risk of bias across all studies, with a JBI score rating of between 87.5 and $100 \%$ (Electronic Supplementary Material 1). A total of eight studies had a low risk of bias, with all JBI items being met. However, two studies [45, 46] had a high risk of bias regarding strategies to deal with confounding factors. Generally, a high risk of bias might also result from the difficulty of randomization and blinding in such clinical trials including patients.

\section{Discussion}

This systematic review and meta-analysis examined the effects of ACL injury on knee proprioception in patients with surgical arthroscopic ACL reconstruction and conservatively treated patients without surgery compared with HCs. The main findings revealed that proprioception after surgical restoration by anatomic ACL reconstruction was significantly lower compared with the control group, while no significant differences were observed when comparing conservative treatment with HCs. For ACLD patients, outcomes for JPS were more clear compared with the TTDPM (with only two available studies and a $p$-value of 0.05 ). These results highlight the substantial proprioceptive consequences of surgical ACL repair that need to be taken into account when discussing individuals' treatment procedures and rehabilitative approaches. 


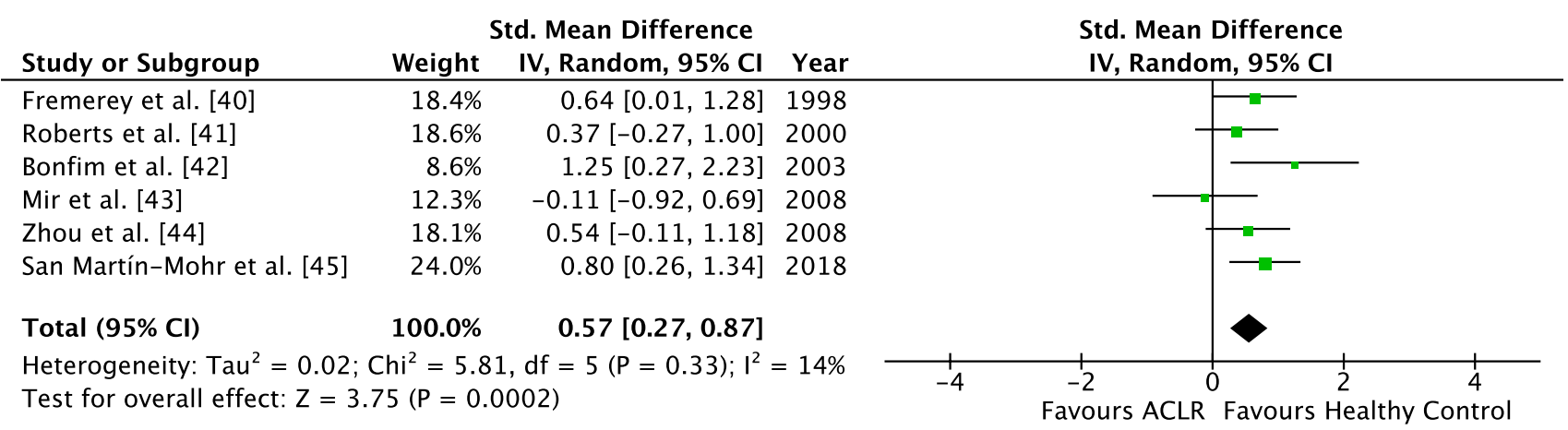

Fig. 2 Forest plot of the ACLR group demonstrating the JPS between the operated group and the healthy control group. ACLR anterior cruciate ligament reconstructed, JPS joint position sense, Std. standardized, $I V$ inverse variance, $C I$ confidence interval, $d f$ degrees of freedom

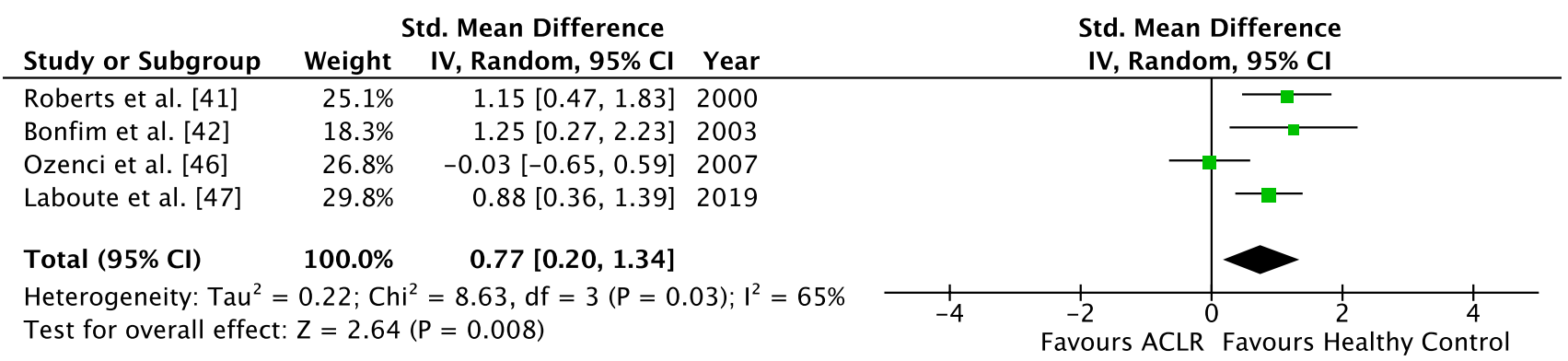

Fig. 3 Forest plot of the ACLR group demonstrating the TTDPM between the operated group and the healthy control group. ACLR anterior cruciate ligament reconstructed, TTDPM threshold to detec- tion of passive motion, Std. standardized, IV inverse variance, $C I$ confidence interval, $d f$ degrees of freedom
Std. Mean Difference

\begin{tabular}{lrrr} 
Study or Subgroup & Weight & IV, Random, 95\% Cl & Year \\
\hline Friden et al. [48] & $25.1 \%$ & $-0.37[-1.04,0.30]$ & 1997 \\
Fremerey et al. [40] & $24.9 \%$ & $1.40[0.70,2.10]$ & 1998 \\
Relph et al. [49] & $24.5 \%$ & $1.98[1.21,2.75]$ & 2016 \\
Zult et al. [50] & $25.5 \%$ & $-0.66[-1.23,-0.08]$ & 2017 \\
& & & \\
Total (95\% Cl) & $\mathbf{1 0 0 . 0 \%}$ & $\mathbf{0 . 5 7}[-\mathbf{0 . 6 9 , 1 . 8 4}]$ &
\end{tabular}

Heterogeneity: $\mathrm{Tau}^{2}=1.54 ; \mathrm{Chi}^{2}=42.13, \mathrm{df}=3(\mathrm{P}<0.00001) ; \mathrm{I}^{2}=93 \%$

Test for overall effect: $Z=0.89(P=0.37)$
Std. Mean Difference IV, Random, $95 \% \mathrm{Cl}$

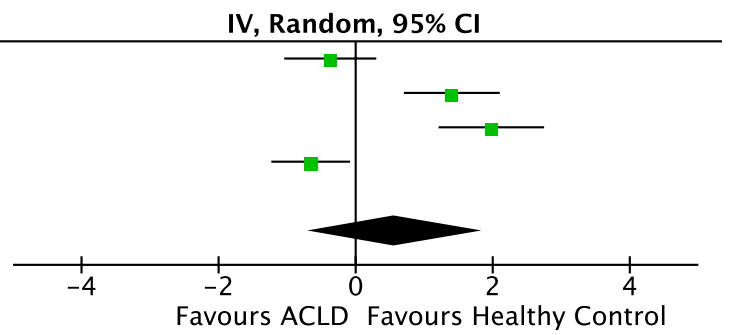

Fig. 4 Forest plot of the ACLD group demonstrating the JPS between the conservatively treated group and the healthy control group. ACLD anterior cruciate ligament deficient, JPS joint position sense, Std. standardized, $I V$ inverse variance, $C I$ confidence interval, $d f$ degrees of freedom

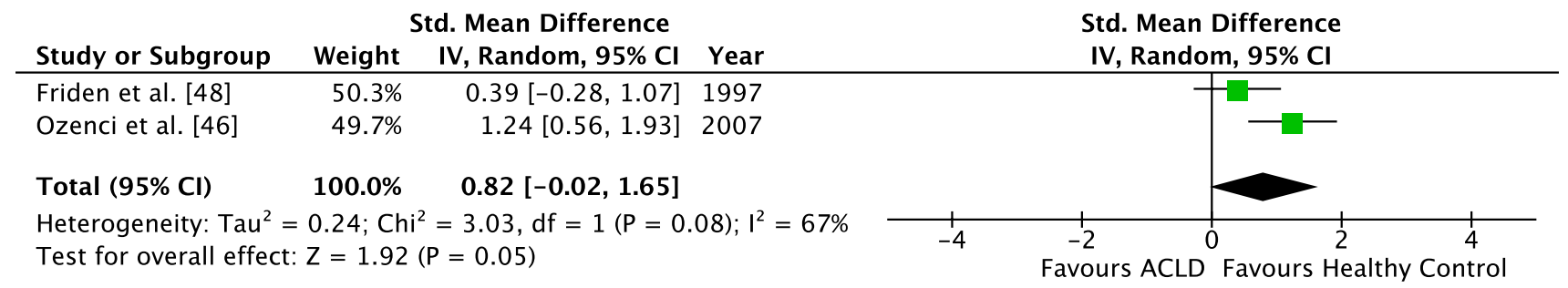

Fig. 5 Forest plot of the ACLD group demonstrating the TTDPM between the conservatively treated group and the healthy control group. $A C L D$ anterior cruciate ligament deficient, TTDPM threshold to detection of passive motion, Std. standardized, $I V$ inverse variance, $C I$ confidence interval, $d f$ degrees of freedom 
Table 5 Quality score of the included studies assessed using the Downs and Black Checklist

\begin{tabular}{ll}
\hline Study & $\begin{array}{l}\text { Downs and Black } \\
\text { Checklist score }\end{array}$ \\
\hline Bonfim et al. [42] & 18 \\
Fremerey et al. [40] & 20 \\
Fridén et al. [48] & 18 \\
Laboute et al. [47] & 18 \\
Mir et al. [43] & 17 \\
Ozenci et al. [46] & 16 \\
Relph et al. [49] & 22 \\
Roberts et al. [41] & 16 \\
San Martín-Mohr et al. [45] & 26 \\
Zhou et al. [44] & 17 \\
Zult et al. [50] & 17 \\
Mean & 19 \\
\hline
\end{tabular}

\subsection{Proprioceptive and Functional Relevance of an ACL Reconstruction}

From a functional perspective, the proprioception of the ACL comprises three major components: (1) a static awareness of the knee joint position; (2) a dynamic detection of knee movement and acceleration; and (3) a closed loop reflex arc, which elicits hamstring reflex responses and regulates synergistic and antagonistic muscle contractions to prevent articular injuries [19, 51, 52]. Adequate sensation of joint movement and joint position in the almost terminal knee position (as observed in this review) is of clinical relevance in conditions when synergistically passive (ligamentous) and active (contractile) restraints to anterior tibial translation or rotational stress are required $[24,53,54]$. The aforementioned conditions entail jumping, pivoting, and landing maneuvers for which scientific evidence suggests that poor proprioception is highly related to motor dysfunction [55, 56] and changes arthrokinematics at articular peak loading in ACL-risky situations [57]. Compromised landing [58] and jumping mechanics $[59,60]$ arise in unilaterally ACL reconstructed patients compared with HCs and are accompanied by neuromuscular deficits in the musculature encompassing the knee joint [61-63], with the result of an elevated risk of ACL recurrences, and meniscal or cartilage injuries [64, 65]. The delayed detection of disadvantageous knee kinematics when the ACL experiences exceeding strain or strain rates (TTDPM) coupled with inaccuracy in sensory joint positioning are key deficits [referring to (1) and (2) above] that expose ACLR patients to re-injuries [9]. Therefore, adequate assessment of knee proprioception in these vulnerable joint angle positions is of exceptional importance. An additional deficit refers to the delayed and diminished contractile responses [referring to (3) above]: knee extensor and flexor forces account, on average, for up to $4500 \mathrm{~N}$ [54] when maximally contracted and therefore serve as important shelters for articular knee structures. In comparison, the ACL only sustains approximately $400 \mathrm{~N}$ [66], which is equivalent to a proportion of $1 / 10$ of muscle force. The commonly known quadriceps and hamstring weaknesses after ACL tears [67] are mediated by reduced muscle activations [67] and may arise partly from non-existent stimulation of group II or III fibers due to the surgical removal of mechanoreceptors (synchronously with the ACL) after torn or arthroscopically reconstructed ACL with auto- and allografts [67]. Analyzing the results of this study with reference to the aforementioned literature, it becomes apparent that proprioceptive deficits account for multiple aspects with clinical consequences.

\subsection{Proprioceptive and Functional Relevance of an ACL Deficiency}

Despite the evidence-based chain of reasoning and functional consequences of an ACL reconstruction, it is under debate why the conservative treatment of an ACL tear is superior compared with ACLR, with regard to proprioception assessed using JPS. The interpretation requires more precautions for TTDPM before an affirmative clinical conclusion can be made as the entire sample size comprises 36 patients only and the level of significance is equal to 0.05 . As a further important aspect, the test sensitivity and reliability (for TTDPM > JPS [68]), as well as the required time to recover full proprioceptive capacity after the ACL tear, needs to be considered.

It is generally acknowledged that native and intact ACLs are equipped with mechanoreceptors that are responsible for proper proprioceptive functioning [69]. These receptor types are sensitive to mechanical deformation and modify neuromuscular function by afferent feedback [69]. Indeed, recent studies have demonstrated that the number of mechanoreceptors in the ACL is positively associated with the accuracy of gold-standard measurements (JPS and TTDPM) of proprioception [51]. This indicates that proprioceptive function and functional stability are closely related to the number of mechanoreceptors in ACLD patients with remnants [70]. Remnants are ACL stumps that remain after ligament rupture and have been shown to possess operating mechanoreceptors that seem to be dependent on injury duration [71] and reliant on continued loading of the remnant [72]. The fact that remnants are still equipped with functioning sensory elements led researchers to speculate about their role in proprioception and knee stability in ACLD populations or patients who underwent a recent fusion surgery with the new tendinous graft attached to the remnant [73]. Although the remnant situation was not reported in the majority of included studies in this systematic review, it might be speculated that patients treated with a conservative approach to 
ACL rehabilitation profit from remaining ACL remnants that again help to preserve knee proprioception, as indicated for the JPS. However, this needs to be further investigated.

\subsection{Rehabilitative Aspects and Considerations for Return to Sports}

Various models and algorithms have been defined that can guide the rehabilitation and decision for return to sport $[74$, 75]. The requirements for high-level sports activity after ACL tears are defined as strength, power, balance, proprioception, speed, and agility [76, 77]. Despite proprioception being key among the primary criteria for functional performance and postural stability, there is no consensus on a method to objectively determine the level of proprioception at the time of return to sport after injury. Although previous research demonstrated remarkable correlations between knee proprioception and dynamic balance $[78,79]$, there is also no consensus about the relative importance of proprioception in the process of decision making for return to sport, especially when considering a net proprioception error of $\leq 2^{\circ}$, as illustrated by the outcomes of the current metaanalysis. Despite the medium to large effect sizes (SMD $0.57-0.82$ ), clinical evidence about the effects of such proprioception deficits on movement kinematics and kinetics is still lacking.

Although not assessed in the current meta-analysis, impaired proprioception in patients with ACLR might facilitate the occurrence of unfavorable knee positions during jumping or landing tasks, which are frequently incorporated in many return to sport protocols $[80,81]$. This hypothesis is supported by recent evidence demonstrating clear relationships between knee proprioception and landing kinematics by showing that individuals with higher levels of proprioception were able to better control knee flexion angles at initial contact during dynamic tasks [56].

Since proprioception itself is greatly mediated by neural pathways, holistic strategies of motor control (being greatly reliant on feedback about body and limb position [10]) are moving increasingly into the focus of the multicomponent assessment for ensuring proper recovery from ACLR [82]. As a potential measure, myoelectrical latency and amplitude assessments are often incorporated in return to sport tasks (i.e. frontal, rotational or lateral jumps, running, landing or cutting) and contain important information about the integration of proprioception in sports and the individual protective strategies for injury avoidance $[83,84]$. These have to be completed under great time pressure and physical exertion.

\subsection{Methodological Considerations}

For an adequate interpretation of the results, potential limitations need to be considered. The current systematic review included only studies that assessed proprioception within a time period of 24 months post injury. Although this might impact the generalizability of our findings to longer-lasting proprioceptive deficits, this approach was intended to ensure homogeneity of included studies. Despite the strict inclusion criteria of the present work, there was still heterogeneity between studies, which might impact the interpretation of the results (especially in the comparison between ACL deficient patients and $\mathrm{HCs}$ ). One potential reason for the observed heterogeneity might be found in the non-standardized protocols for the evaluation of proprioception, including patients' position (lying, sitting, standing), target angles $\left(10-100^{\circ}\right)$, direction of movements (flexion, extension) and techniques (dynamometer, goniometer, analog scale). To address this heterogeneity, we a priori defined strict eligibility and prioritization criteria. Finally, further research needs to evaluate to what extent these differences lead to functional differences between patients with and without surgical treatment of ACL and HCs. Although the present study focused on studies within a range of 24 months post treatment, it might be speculated that time-dependent changes in proprioception deficits occur for both ACLD and ACLR knees caused by histological healing processes. Since this analysis was not possible within the current design due to limited study availability, future studies are warranted that further investigate this research question.

\section{Conclusion and Perspectives}

The findings from the present systematic review and metaanalysis indicate that proprioceptive deficits are present after operative treatment of ACL. No significant deficits were detected following conservative treatment for JPS; however, greater caution is required in relation to interpretation of TTDPM and drawing an affirmative clinical conclusion. These results are of major importance for clinicians and surgeons when planning an individualized ACL rehabilitation approach. In this context, further research is needed in order to examine the mechanisms underlying the differences for treatment approaches as well as functional relevance and consequences of impaired proprioception in ACL patients. Recent technical progress in ACL surgeries suggests that ACL augmentation using remnant-preserving ACL reconstruction may produce satisfactory clinical outcomes because preservation of the ACL remnant can be beneficial in terms of proprioception, biomechanical functions, and vascularization of the graft. With an emphasis on novel surgical approaches and the duration of healing and rehabilitation periods [85], the interrelationship between surgical reconstruction and the preservation of proprioception needs further elaboration. 
Supplementary Information The online version contains supplementary material available at https://doi.org/10.1007/s40279-021-01600-z.

\section{Declarations}

Funding No sources of funding were used to assist in the preparation of this article.

Conflict of interest John Dick Fleming, Ramona Ritzmann and Christoph Centner declare that they have no conflicts of interest relevant to the content of this review.

Availability of data and material The datasets generated and analyzed during the current systematic review and meta-analysis are available from the corresponding author upon reasonable request.

Code availability Not applicable.

Author contributions JF, RR and CC conceived the study. JF and CC completed the study protocol and registration. JF, RR and CC completed the database searches and extraction. JF and CC completed the title and abstract screening. JF and CC completed the full-text review. $\mathrm{JF}$ and $\mathrm{CC}$ completed the risk-of-bias assessment. JF and CC completed the data extraction. JF and CC completed the data analysis. JF drafted the Introduction and Methods sections, and RR and CC drafted the Results and Discussion sections. All authors reviewed, revised and approved the final manuscript.

Open Access This article is licensed under a Creative Commons Attribution 4.0 International License, which permits use, sharing, adaptation, distribution and reproduction in any medium or format, as long as you give appropriate credit to the original author(s) and the source, provide a link to the Creative Commons licence, and indicate if changes were made. The images or other third party material in this article are included in the article's Creative Commons licence, unless indicated otherwise in a credit line to the material. If material is not included in the article's Creative Commons licence and your intended use is not permitted by statutory regulation or exceeds the permitted use, you will need to obtain permission directly from the copyright holder. To view a copy of this licence, visit http://creativecommons.org/licenses/by/4.0/.

\section{References}

1. Petersen W, Zantop T, Becker R. Das vordere Kreuzband. Grundlagen und aktuelle Praxis der operativen Therapie. Cologne: Deutscher Ärzte-Verl; 2009.

2. Freeman MA, Wyke B. The innervation of the knee joint. An anatomical and histological study in the cat. J Anat. 1967;101(Pt 3):505-32.

3. Dyhre-Poulsen P, Krogsgaard MR. Muscular reflexes elicited by electrical stimulation of the anterior cruciate ligament in humans. J Appl Physiol. 2000;89(6):2191-5.

4. Freeman MA, Wyke B. Articular reflexes at the ankle joint: an electromyographic study of normal and abnormal influences of ankle-joint mechanoreceptors upon reflex activity in the leg muscles. Br J Surg. 1967;54(12):990-1001.

5. Grigg P, Schaible HG, Schmidt RF. Mechanical sensitivity of group III and IV afferents from posterior articular nerve in normal and inflamed cat knee. J Neurophysiol. 1986;55(4):635-43.

6. Ardern CL, Taylor NF, Feller JA, Webster KE. Fifty-five per cent return to competitive sport following anterior cruciate ligament reconstruction surgery: an updated systematic review and metaanalysis including aspects of physical functioning and contextual factors. Br J Sports Med. 2014;48(21):1543-52.

7. Paterno MV, Rauh MJ, Schmitt LC, Ford KR, Hewett TE. Incidence of second ACL injuries 2 years after primary ACL reconstruction and return to sport. Am J Sports Med. 2014;42(7):1567-73.

8. Cinque ME, Dornan GJ, Chahla J, Moatshe G, LaPrade RF High rates of osteoarthritis develop after anterior cruciate ligament surgery: an analysis of 4108 patients. Am J Sports Med. 2018;46(8):2011-9.

9. Lephart E, Fu FH. Proprioception and neuromuscular control in joint stability. Percept Motor Skill. 2001;92(1):319-20.

10. Riemann BL, Lephart SM. The sensorimotor system, part I: the physiologic basis of functional joint stability. J Athl Train. 2002;37(1):71-9.

11. Liu-Ambrose $\mathrm{T}$. The anterior cruciate ligament and functional stability of the knee joint. B C Med J. 2003;45(10):5.

12. Young SW, Valladares RD, Loi F, Dragoo JL. Mechanoreceptor reinnervation of autografts versus allografts after anterior cruciate ligament reconstruction. Orthop J Sports Med. 2016;4(10):2325967116668782.

13. Proske U, Gandevia SC. The proprioceptive senses: their roles in signaling body shape, body position and movement, and muscle force. Physiol Rev. 2012;92(4):1651-97.

14. Smith TO, Davies L, Hing CB. A systematic review to determine the reliability of knee joint position sense assessment measures. Knee. 2013;20(3):162-9.

15. Riemann BL, Myers JB, Lephart SM. Sensorimotor system measurement techniques. J Athl Train. 2002;37(1):85-98.

16. Boerboom AL, Huizinga MR, Kaan WA, Stewart RE, Hof AL, Bulstra SK, et al. Validation of a method to measure the proprioception of the knee. Gait Posture. 2008;28(4):610-4.

17. Relph N, Herrington L, Tyson S. The effects of ACL injury on knee proprioception: a meta-analysis. Physiotherapy. 2014;100(3):187-95.

18. Kim HJ, Lee JH, Lee DH. Proprioception in patients with anterior cruciate ligament tears a meta-analysis comparing injured and uninjured limbs. Am J Sports Med. 2017;45(12):2916-22.

19. Grigg P. Mechanical factors influencing response of joint afferent neurons from cat knee. J Neurophysiol. 1975;38(6):1473-84.

20. Grigg P. Response of joint afferent neurons in cat medial articular nerve to active and passive movements of the knee. Brain Res. 1976;118(3):482-5.

21. Moher D, Liberati A, Tetzlaff J, Altman DG, Group P. Preferred reporting items for systematic reviews and meta-analyses: the PRISMA statement. Ann Intern Med. 2009;151(4):264-9 (W64).

22. Horsley T, Dingwall O, Sampson M. Checking reference lists to find additional studies for systematic reviews. Cochrane Database Syst Rev. 2011;2011(8):MR000026.

23. Arockiaraj J, Korula RJ, Oommen AT, Devasahayam S, Wankhar $\mathrm{S}$, Velkumar S, et al. Proprioceptive changes in the contralateral knee joint following anterior cruciate injury. Bone Jt J. 2013;95-B(2):188-91.

24. Markolf KL, Burchfield DI, Shapiro MM, Shepard ME, Finerman GAM, Slauterbeck JL. Combined knee loading states that generate high anterior cruciate ligament forces. J Orthop Res. 1995;13(6):930-5.

25. Li G, Rudy TW, Sakane M, Kanamori A, Ma CB, Woo SL. The importance of quadriceps and hamstring muscle loading on knee kinematics and in-situ forces in the ACL. J Biomech. 1999;32(4):395-400.

26. Iwasa J, Ochi M, Uchio Y, Adachi N, Kawasaki K. Decrease in anterior knee laxity by electrical stimulation of normal and 
reconstructed anterior cruciate ligaments. J Bone Jt Surg Br. 2006;88(4):477-83.

27. Hoshiba T, Nakata H, Saho Y, Kanosue K, Fukubayashi T. Comparison of the position-matching and position-reproducing tasks to detect deficits in knee position sense after reconstruction of the anterior cruciate ligament. J Sport Rehabil. 2020;29(1):87-92.

28. Downs SH, Black N. The feasibility of creating a checklist for the assessment of the methodological quality both of randomised and non-randomised studies of health care interventions. J Epidemiol Commun Health. 1998;52(6):377-84.

29. Gronfeldt BM, Lindberg Nielsen J, Mieritz RM, Lund H, Aagaard P. Effect of blood-flow restricted vs. heavy-load strength training on muscle strength: systematic review and meta-analysis. Scand J Med Sci Sports. 2020;30(5):837-48.

30. Centner C, Lauber B. A systematic review and meta-analysis on neural adaptations following blood flow restriction training: what we know and what we don't know. Front Physiol. 2020;11:887.

31. Olsen OE, Myklebust G, Engebretsen L, Bahr R. Injury mechanisms for anterior cruciate ligament injuries in team handball a systematic video analysis. Am J Sports Med. 2004;32(4):1002-12.

32. Krosshaug T, Nakamae A, Boden BP, Engebretsen L, Smith G, Slauterbeck JR, et al. Mechanisms of anterior cruciate ligament injury in basketball-video analysis of 39 cases. Am J Sports Med. 2007;35(3):359-67.

33. Mehl J, Diermeier T, Herbst E, Imhoff AB, Stoffels T, Zantop $\mathrm{T}$, et al. Evidence-based concepts for prevention of knee and ACL injuries 2017 guidelines of the ligament committee of the German Knee Society (DKG). Arch Orthop Trauma Surg. 2018;138(1):51-61.

34. Silverman SR, Schertz LA, Yuen HK, Lowman JD, Bickel CS. Systematic review of the methodological quality and outcome measures utilized in exercise interventions for adults with spinal cord injury. Spinal Cord. 2012;50(10):718-27.

35. Moola S, Munn Z, Tufanaru C, Aromataris E, Sears K, Sfetcu R, et al. Chapter 7: systematic reviews of etiology and risk. In: Aromataris E, Munn Z, editors. JBI Manual for Evidence Synthesis: JBI; 2020.

36. Ma L-L, Wang Y-Y, Yang Z-H, Huang D, Weng H, Zeng X-T. Methodological quality (risk of bias) assessment tools for primary and secondary medical studies: what are they and which is better? Mil Med Res. 2020;7(1):7.

37. Martins JNR, Marques D, Leal Silva EJN, Caramês J, Mata A, Versiani MA. Influence of demographic factors on the prevalence of a second root canal in mandibular anterior teeth-a systematic review and meta-analysis of cross-sectional studies using cone beam computed tomography. Arch Oral Biol. 2020;116:104749.

38. Melo G, Dutra KL, Rodrigues Filho R, Ortega AOL, Porporatti AL, Dick B, et al. Association between psychotropic medications and presence of sleep bruxism: a systematic review. J Oral Rehabil. 2018;45(7):545-54.

39. Higgins JPT, Green S. Cochrane Handbook for Systematic Reviews of Interventions. Version 5.1.0 [updated March 2011]. The Cochrane Collaboration; 2011.

40. Fremerey RW, Lobenhoffer P, Born I, Tscherne H, Bosch U. Can knee joint proprioception by reconstruction of the anterior cruciate ligament be restored? A prospective longitudinal study [in German]. Unfallchirurg. 1998;101(9):697-703.

41. Roberts D, Friden T, Stomberg A, Lindstrand A, Moritz U. Bilateral proprioceptive defects in patients with a unilateral anterior cruciate ligament reconstruction: a comparison between patients and healthy individuals. J Orthop Res. 2000;18(4):565-71.

42. Bonfim TR, Jansen Paccola CA, Barela JA. Proprioceptive and behavior impairments in individuals with anterior cruciate ligament reconstructed knees. Arch Phys Med Rehabil. 2003;84(8):1217-23.
43. Mir SM, Hadian MR, Talebian S, Nasseri N. Functional assessment of knee joint position sense following anterior cruciate ligament reconstruction. Br J Sports Med. 2008;42(4):300-3.

44. Zhou MW, Gu L, Chen YP, Yu CL, Ao YF, Huang HS, et al. Factors affecting proprioceptive recovery after anterior cruciate ligament reconstruction. Chin Med J (Engl). 2008;121(22):2224-8.

45. San Martín-Mohr C, Cristi-Sanchez I, Pincheira PA, Reyes A, Berral FJ, Oyarzo C. Knee sensorimotor control following anterior cruciate ligament reconstruction: a comparison between reconstruction techniques. PLoS ONE. 2018;13(11):e0205658.

46. Ozenci AM, Inanmaz E, Ozcanli H, Soyuncu Y, Samanci N, Dagseven T, et al. Proprioceptive comparison of allograft and autograft anterior cruciate ligament reconstructions. Knee Surg Sports Traumatol Arthrosc. 2007;15(12):1432-7.

47. Laboute E, Verhaeghe E, Ucay O, Minden A. Evaluation kinaesthetic proprioceptive deficit after knee anterior cruciate ligament (ACL) reconstruction in athletes. J Exp Orthop. 2019;6(1):6.

48. Fridén T, Roberts D, Zatterstrom R, Lindstrand A, Moritz U. Proprioception after an acute knee ligament injury: a longitudinal study on 16 consecutive patients. J Orthop Res. 1997;15(5):637-44.

49. Relph N, Herrington L. Knee joint position sense ability in elite athletes who have returned to international level play following ACL reconstruction: a cross-sectional study. Knee. 2016;23(6):1029-34.

50. Zult T, Gokeler A, van Raay JJ, Brouwer RW, Zijdewind I, Hortobagyi T. An anterior cruciate ligament injury does not affect the neuromuscular function of the non-injured leg except for dynamic balance and voluntary quadriceps activation. Knee Surg Sports Traumatol Arthrosc. 2017;25(1):172-83.

51. Adachi N, Ochi M, Uchio Y, Iwasa J, Ryoke K, Kuriwaka M. Mechanoreceptors in the anterior cruciate ligament contribute to the joint position sense. Acta Orthop Scand. 2002;73(3):330-4.

52. Johansson H. Role of knee ligaments in proprioception and regulation of muscle stiffness. J Electromyogr Kinesiol. 1991;1(3):158-79.

53. Renstrom P, Arms SW, Stanwyck TS, Johnson RJ, Pope MH. Strain within the anterior cruciate ligament during hamstring and quadriceps activity. Am J Sports Med. 1986;14(1):83-7.

54. DeMorat G, Weinhold P, Blackburn T, Chudik S, Garrett W. Aggressive quadriceps loading can induce noncontact anterior cruciate ligament injury. Am J Sports Med. 2004;32(2):477-83.

55. Barrett DS. Proprioception and function after anterior cruciate reconstruction. J Bone Jt Surg Br. 1991;73(5):833-7.

56. Nagai T, Sell TC, House AJ, Abt JP, Lephart SM. Knee proprioception and strength and landing kinematics during a single-leg stop-jump task. J Athl Train. 2013;48(1):31-8.

57. Yang C, Tashiro Y, Lynch A, Fu F, Anderst W. Kinematics and arthrokinematics in the chronic ACL-deficient knee are altered even in the absence of instability symptoms. Knee Surg Sport Traumatol Arthrosc. 2018;26(5):1406-13.

58. Bell DR, Smith MD, Pennuto AP, Stiffler MR, Olson ME. Jump-landing mechanics after anterior cruciate ligament reconstruction: a landing error scoring system study. J Athl Train. 2014;49(4):435-41.

59. Delahunt E, Sweeney L, Chawke M, Kelleher J, Murphy K, Patterson $\mathrm{M}$, et al. Lower limb kinematic alterations during drop vertical jumps in female athletes who have undergone anterior cruciate ligament reconstruction. J Orthop Res. 2012;30(1):72-8.

60. Kuenze CM, Foot N, Saliba SA, Hart JM. Drop-landing performance and knee-extension strength after anterior cruciate ligament reconstruction. J Athl Train. 2015;50(6):596-602.

61. Vairo GL, Myers JB, Sell TC, Fu FH, Harner CD, Lephart SM. Neuromuscular and biomechanical landing performance 
subsequent to ipsilateral semitendinosus and gracilis autograft anterior cruciate ligament reconstruction. Knee Surg Sport Tr A. 2008;16(1):2-14.

62. Di Stasi S, Myer GD, Hewett TE. Neuromuscular training to target deficits associated with second anterior cruciate ligament injury. J Orthop Sports Phys Ther. 2013;43(11):777-92 (A1-11).

63. Nyland J, Gamble C, Franklin T, Caborn DNM. Permanent knee sensorimotor system changes following ACL injury and surgery. Knee Surg Sport Tr A. 2017;25(5):1461-74.

64. Paterno MV, Schmitt LC, Ford KR, Rauh MJ, Myer GD, Huang B, et al. Biomechanical measures during landing and postural stability predict second anterior cruciate ligament injury after anterior cruciate ligament reconstruction and return to sport. Am J Sports Med. 2010;38(10):1968-78.

65. Paterno MV, Huang B, Thomas S, Hewett TE, Schmitt LC. Clinical factors that predict a second ACL injury after ACL reconstruction and return to sport preliminary development of a clinical decision algorithm. Orthop J Sports Med. 2017;5(12):2325967117745279.

66. Paschos NK, Gartzonikas D, Barkoula N-M, Moraiti C, Paipetis A, Matikas TE, et al. Cadaveric study of anterior cruciate ligament failure patterns under uniaxial tension along the ligament. Arthroscopy. 2010;26(7):957-67.

67. Konishi Y, Fukubayashi T, Takeshita D. Possible mechanism of quadriceps femoris weakness in patients with ruptured anterior cruciate ligament. Med Sci Sport Exerc. 2002;34(9):1414-8.

68. Reider B, Arcand MA, Diehl LH, Mroczek K, Abulencia A, Stroud $\mathrm{CC}$, et al. Proprioception of the knee before and after anterior cruciate ligament reconstruction. Arthroscopy. 2003;19(1):2-12.

69. Ergen E, Ulkar B. Chapter 18-proprioception and coordination. In: Frontera WR, Herring SA, Micheli LJ, Silver JK, Young TP, editors. Clinical sports medicine. Edinburgh: W.B. Saunders; 2007. p. 237-55.

70. Dhillon MS, Bali K, Prabhakar S. Differences among mechanoreceptors in healthy and injured anterior cruciate ligaments and their clinical importance. Muscles Ligaments Tendons J. 2012;2(1):38-43.

71. Dhillon MS, Bali K, Vasistha RK. Immunohistological evaluation of proprioceptive potential of the residual stump of injured anterior cruciate ligaments (ACL). Int Orthop. 2010;34(5):737-41.

72. Kosy JD, Mandalia VI. Anterior cruciate ligament mechanoreceptors and their potential importance in remnant-preserving reconstruction: a review of basic science and clinical findings. J Knee Surg. 2018;31(8):736-46.

73. Nakase J, Toratani T, Kosaka M, Ohashi Y, Tsuchiya H. Roles of ACL remnants in knee stability. Knee Surg Sport Traumatol Arthrosc. 2013;21(9):2101-6.

74. Albano TR, Rodrigues CAS, Melo AKP, de Paula PO, Almeida GPL. Clinical decision algorithm associated with return to sport after anterior cruciate ligament reconstruction. J Athl Train. 2020;55(7):691-8.

75. Jordan MJ, Morris N, Lane M, Barnert J, MacGregor K, Heard M, et al. Monitoring the return to sport transition after ACL injury: an alpine ski racing case study. Front Sports Act Living. 2020;2:12.

76. Herbst E, Hoser C, Hildebrandt C, Raschner C, Hepperger C, Pointner $\mathrm{H}$, et al. Functional assessments for decision-making regarding return to sports following ACL reconstruction. Part II: clinical application of a new test battery. Knee Surg Sports Traumatol Arthrosc. 2015;23(5):1283-91.

77. Hildebrandt C, Müller L, Zisch B, Huber R, Fink C, Raschner C. Functional assessments for decision-making regarding return to sports following ACL reconstruction. Part I: development of a new test battery. Knee Surg Sports Traumatol Arthrosc. 2015;23(5):1273-81.

78. Lee HM, Cheng CK, Liau JJ. Correlation between proprioception, muscle strength, knee laxity, and dynamic standing balance in patients with chronic anterior cruciate ligament deficiency. Knee. 2009;16(5):387-91

79. Wang H, Ji Z, Jiang G, Liu W, Jiao X. Correlation among proprioception, muscle strength, and balance. J Phys Ther Sci. 2016;28(12):3468-72.

80. Kotsifaki A, Whiteley R, Van Rossom S, Korakakis V, Bahr R, Sideris V, et al. Single leg hop for distance symmetry masks lower limb biomechanics: time to discuss hop distance as decision criterion for return to sport after ACL reconstruction? Br J Sports Med. 2021:bjsports-2020-103677.

81. Lambert C, Lambert M, Ellermann A, Wafaisade A, Buse C, Peters N, et al. Development of a modified cross-over hop test to reduce measurement errors in return-to-competition testing. Sportverletz Sportschaden. 2021. https://doi.org/10.1055/a-1349-5605.

82. Rocchi JE, Labanca L, Laudani L, Minganti C, Mariani PP, Macaluso A. Timing of muscle activation is altered during singleleg landing tasks after anterior cruciate ligament reconstruction at the time of return to sport. Clin J Sport Med. 2020;30(6):e186-93.

83. Konishi Y, McNair PJ, Rice DA, Ochiai S, Hagino T. Stretch reflex changes in ACL-deficient individuals and healthy controls during normal and surprise landings. Scand J Med Sci Sports. 2020;30(12):2342-51.

84. Haines S, Baker T, Donaldson M. Development of a physical performance assessment checklist for athletes who sustained a lower extremity injury in preparation for return to sport: a Delphi study. Int J Sports Phys Ther. 2013;8(1):44-53.

85. Muneta T, Koga H. Anterior cruciate ligament remnant and its values for preservation. Asia Pac J Sports Med Arthrosc Rehabilit Technol. 2017;7:1-9. 